\title{
COMPARATIVE STUDY BETWEEN INTRAVENOUS DEXMEDETOMIDINE AND ESMOLOL IN ATTENUATION OF HAEMODYNAMIC STRESS RESPONSE DURING ENDOTRACHEAL INTUBATION AND LARYNGOSCOPY
}

\author{
Tshering P. Bhutia1 ${ }^{1}$ Purvi J. Mehta ${ }^{2}$, Sugadev 3
}

${ }_{13}^{\text {rd }}$ Year Resident, Department of Anaesthesiology and Critical Care, M. P. Government Medical College, GG Hospital, Gujarat, India. ${ }^{2}$ Associate Professor, Department of Anaesthesiology and Critical Care, M. P. Government Medical College, GG Hospital, Gujarat, India. 32nd Year Resident, Department of Anaesthesiology and Critical Care, M. P. Government Medical College, GG Hospital, Gujarat, India.

\section{ABSTRACT}

\section{BACKGROUND}

Laryngoscopy and endotracheal intubation produce sympathetic overdrive by catecholamine release resulting in hypertension and tachycardia. Various agents are being tried to combat the intubation response.

The aim of this study is to compare the efficacy of dexmedetomidine which is a highly selective alpha-2 agonist with an ultrashort-acting beta blocker esmolol in attenuating the haemodynamic stress response secondary to laryngoscopy and endotracheal intubation.

\section{MATERIALS AND METHODS}

After obtaining an approval from Institutional Ethics Committee and after having informed and written consent from each patient, 60 adult patients scheduled for elective surgery under general anaesthesia were selected and were provided general anaesthesia with endotracheal intubation for all patients. Patients were randomly allocated into two groups, Group E and Group D with 30 cases in each group. Group D - Received Dexmedetomidine $1 \mathrm{mcg} / \mathrm{kg}$, Group E - Received Esmolol 0.5 mg/kg. Patient's HR, SBP, DBP and MAP was recorded at baseline, after infusion of study drug, after induction of anaesthesia, immediately and 1, 3, 5 and 7 minutes after intubation. All the study parameters were collected and documented by a single anaesthesiologist in all cases, who was blinded for the content in the syringe and study protocol.

\section{RESULTS}

The percentage change of all haemodynamic parameters from baseline were less in the dexmedetomidine group than in esmolol group at all time points of measurement. However, a statistically significant difference was observed often at time points within 1 minute and 3 minutes after tracheal intubation.

\section{CONCLUSION}

The incidence of hypertension and tachycardia following laryngoscopy and intubation were significantly lower in patients receiving intravenous dexmedetomidine infusion when compared with patients receiving intravenous esmolol infusion.

\section{KEY WORDS}

Dexmedetomidine, Esmolol, Haemodynamics, Intubation, Laryngoscopy.

HOW TO CITE THIS ARTICLE: Bhutia TP, Mehta PJ, Sugadev. Comparative study between intravenous dexmedetomidine and esmolol in attenuation of haemodynamic stress response during endotracheal intubation and laryngoscopy. J. Evolution Med. Dent. Sci. 2018;7(44):4770-4776, DOI: 10.14260/jemds/2018/1064

\section{BACKGROUND}

Laryngoscopy and tracheal intubation have become an integral part of anaesthetic management and critical care since their description in 1921 by Rowbotham and Magill. ${ }^{1}$ Circulatory response to laryngeal and tracheal stimulation in the form of tachycardia, hypertension and dysrhythmia was known since 1940.1,2 The principle mechanism behind the hypertension and tachycardia is the exaggerated sympathetic action due to increased catecholamine release. ${ }^{3}$ The increase in heart rate and blood pressure is usually transient, variable and unpredictable.

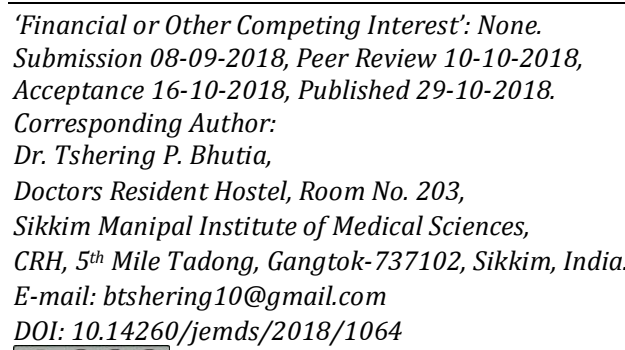

It may not be of much significance in healthy individuals, but can be hazardous in those with hypertension, cardiac dysfunction, coronary artery disease or cerebrovascular disease. ${ }^{4}$ Laryngoscopic response in such individuals can precipitate coronary insufficiency, pulmonary oedema, arrhythmias, left ventricular failure and cerebrovascular haemorrhage. Dexmedetomidine,5,6,7 the pharmacologically active d-isomer of medetomidine $(4, \quad[5]-[1-(2,3-$ dimethylphenyl)-ethyl] imidazole is a highly specific and selective alpha-2 adrenoreceptor agonist. In recent studies, dexmedetomidine was shown to have clinically significant effects on anaesthetic requirement and haemodynamic responses induced by anaesthesia and surgery in patients.

Since tachycardia appears to be associated more frequently with myocardial ischaemia than does hypertension, an interesting approach towards attenuating cardiac responses to laryngeal stimulation is the use of beta-adrenergic antagonists. Among the beta-adrenergic antagonists, Esmolol8,9,10,11 Methyl 3-\{4-[(2S)-2-hydroxy-3-(isopropyl amino) propoxy] phenyl\} propanoate is an effective option, because it is ultra-short-acting and can be administered intravenously. Thus, this short acting beta-blocker appears 
quite suitable for use during a short-lived stress such as tracheal intubation. Apart from its effect, it is also known to reduce the requirement for anaesthetic agents. ${ }^{8}$

\section{MATERIALS AND METHODS}

It is a prospective, randomised, double-blinded, controlled study. After obtaining an approval from Institutional Ethics Committee (IEC approval number-IEC/123/2016, DATE-2309-2016) and after having informed and written consent from each patient, the sample size of 60 adult patients scheduled for elective surgery under general anaesthesia were taken for convenience. All the selected patients were provided general anaesthesia with endotracheal intubation. Patients were randomly allocated into two groups as Group D and Group E by computer generated numbers. Group E and Group D with 30 cases in each group. Group D - Received Dexmedetomidine one microgram/ kilogram diluted in 20 millilitre $(\mathrm{mL})$ of normal saline infused intravenously over ten minutes before induction and a bolus of $10 \mathrm{~mL}$ of normal saline given slowly (over 30 seconds) intravenously 2 minutes before intubation. Group E - Received $20 \mathrm{~mL}$ of normal saline infused intravenously over 10 minutes before induction and a bolus of Esmolol 0.5 milligram/kilogram diluted in $10 \mathrm{~mL}$ of normal saline given slowly (over 30 seconds) intravenously two minutes before intubation. This was done by a separate anaesthesiologist who was not aware of the study protocol and was not involved in administering drug or data collection during the study. He was not aware of the group allocated to them.

Patient's Heart Rate (HR), non-invasive blood pressure systolic (SBP), diastolic (DBP), Mean Arterial Pressure (MAP) was recorded at baseline after infusion of study drug, after induction of anaesthesia, immediately and one, three, five and seven minutes after intubation. All the study parameters collected and documented by a single anaesthesiologist in all cases, who is blinded for the content in the syringe and study protocol.

Patients undergoing various elective general surgical and gynaecological procedures with ASA physical status 1 and 2 were selected randomly.

Patients with heart disease, hypertension, diabetes mellitus, thyroid abnormalities and those on treatment with cardioactive or inhibitory drugs were excluded from the study. Patients with an anticipated difficult airway and those in whom tracheal intubation took more than 20 seconds and more than one attempt were also excluded from the study.

\section{Patient Preparation}

The procedure was explained to the patient beforehand.

After arrival of the patients to the operating room, patients were connected to monitor (Schiller Truscope2 monitor), ECG and heart rate were monitored continuously, and non-invasive recording of systolic, diastolic and mean arterial pressure were started. An intravenous line was secured in patients, either forearm using 18-gauge intravenous cannula.

All the patients were pre-medicated with intravenous injection of Glycopyrrolate (4 mcg/ $\mathrm{kg}$ ), Ondansetron (80 $\mathrm{mcg} / \mathrm{kg}$ ), Midazolam (20 mcg/kg).

Prior to infusion of study drug HR, SBP, DBP and MAP were recorded and was designated as baseline parameter.

\section{Group D}

Received Dexmedetomidine $1 \mathrm{mcg} / \mathrm{kg}$ diluted in $20 \mathrm{~mL}$ of normal saline infused intravenously over 10 minutes before induction and a bolus of $10 \mathrm{~mL}$ of normal saline given slowly (over 30 seconds) intravenously 2 minutes before intubation.

\section{Group E}

Received $20 \mathrm{~mL}$ of normal saline infused intravenously over 10 minutes before induction and a bolus of Esmolol 0.5 $\mathrm{mg} / \mathrm{kg}$ diluted in $10 \mathrm{~mL}$ of normal saline given slowly (over 30 seconds) intravenously 2 minutes before intubation.

The study drugs were loaded by anaesthesiologist who was not aware of the study protocol and not involved in the recording of study parameters or performance of laryngoscopy.

The patients were then pre-oxygenated with 100\% oxygen for 5 minutes before induction with a properly fitting face mask. Anaesthesia was induced with intravenous injection of propofol at a dose of $2 \mathrm{mg} / \mathrm{kg}$ administered slowly till the end point, i.e. loss of verbal contact. Injection succinylcholine was administered at a dose of $1.5 \mathrm{mg} / \mathrm{kg}$ body weight intravenously.

\section{Procedure}

Direct laryngoscopy was done using rigid laryngoscopy with standard Macintosh blade. Intubation was done with appropriate size disposable, high volume low pressure cuffed endotracheal tube. After intubation, anaesthesia was maintained with oxygen: nitrous (1:3) and volatile anaesthetics with a tidal volume of $8 \mathrm{~mL} / \mathrm{kg}$ and a rate of 12 16 breaths per minute. For maintenance of relaxation, injection, Atracurium was administered according to body weight. Hypoxia and hypercarbia were avoided in all the cases, by keeping the tidal volume constant $(8 \mathrm{~mL} / \mathrm{kg})$ and respiratory rate in a narrow range variation $(12-16 / \mathrm{min})$ to keep the end-tidal carbon-dioxide within 30 - $45 \mathrm{mmHg}$.

\section{Plan of Study}

Patient's heart rate, non-invasive blood pressure (systolic, diastolic, mean arterial pressure) was recorded at baseline, after infusion of study drug, after induction of anaesthesia, immediately and 1, 3, 5 and 7 minutes after intubation. All the study parameters collected and documented by a single anaesthesiologist in all cases, who is blinded for the content in the syringe and study protocol.

Duration of laryngoscopy was defined in this study as the time starting from the introduction of laryngoscopic blade into the oropharynx upto the appearance of the EtCO2 curve on monitor. Duration of laryngoscopy was monitored by a separate anaesthesiologist, who is blinded for the study protocol.

\section{We had the following Terminologies used in our Study}

Significant hypotension was defined in this study as SBP $<25 \%$ of baseline value.

Significant hypertension was defined as SBP $>20 \%$ of baseline value.

Significant tachycardia was defined as HR $>20 \%$ of baseline value.

Significant bradycardia was defined as $\mathrm{HR}<60 /$ minute. 
Mean arterial pressure was calculated by the formula: $\mathrm{MAP}=1 / 3(\mathrm{SBP}+2 \mathrm{DBP})$

One intubation attempt defined as an act of introducing laryngoscope blade between incisors into the oropharynx to achieve endotracheal intubation. If it is removed from the oropharynx for any reason without achieving endotracheal intubation and reinserted it is considered as another attempt and were excluded from the study.

The incidence of hypertension, hypotension, tachycardia and bradycardia between the two groups were recorded.

\section{Statistical Analysis}

Data was compiled in Microsoft Excel worksheet. Mean and standard deviation for all values were calculated and compared within the group, with the baseline values as well as intergroup comparison were done. Paired and unpaired ttests and chi-square test were used for statistical analysis. GraphPad InStat Software was used for performing statistical analysis. $\mathrm{P}<0.05$ was considered significant.

\section{RESULTS}

A total of 60 patients were enrolled in the study with 30 in each group. The difference in the percentage change of MAP from the baseline at $10 \mathrm{~s}$ after intubation between the two groups was used to calculate the power of the study. Power of the study with 30 as size in each sample is $91 \%$. So the sample size was adequate. The demographic profile was comparable in two groups.

The mean baseline HR, SBP, DBP and MAP are comparable in both the groups. Statistical difference being insignificant $(p>0.05)$. After giving the study drug, the mean heart rate decreased by $10.31 \%$ from baseline in Group E. In Group D mean heart rate decreased by $5.37 \%$ after infusion of study drug, which was still comparable to baseline ( $p>$ 0.05).

At 1 minute after laryngoscopy and intubation, heart rates were increased in both groups from baseline. In Group $E$ the increase in heart rate was $7.66 \%$ from the baseline, which was statistically significant $(\mathrm{p}<0.05)$. In Group D the increase in heart rate from baseline was $1.90 \%$, which was statistically insignificant. When both groups were compared, the increase in mean heart rate after laryngoscopy and intubation was found to be significantly high in Group E than in Group D ( $\mathrm{p}<0.05)$.

At 3 minutes after intubation the mean heart rate remained significantly higher in Group E when compared to baseline: the change being $10.22 \%$, which is of statistical significance $(p<0.05)$. There was a decrease in mean heart rate $(2.93 \%)$ from baseline in Group D, which was statistically insignificant $(p>0.05)$. When both the groups were compared the increase in mean heart rate was higher in Group E, which was highly significant $(\mathrm{p}<0.001)$.

In Group E, the mean heart rate remained higher than baseline at 5 minutes after intubation, but was statistically insignificant ( $p>0.05$ ). In Group D, there was a significant decrease in mean heart rate from baseline at 5 minutes after intubation $(p<0.05)$. There was highly significant difference in the mean heart rate between the groups at 5 minutes after intubation.

In Group E, increase in mean heart rate produced by laryngoscopy and intubation returned to near baseline at 7 minutes after intubation ( $p>0.05)$. In Group D, there was $10.98 \%$ decrease in heart rate from baseline at 7 minutes after intubation: statistical difference being highly significant ( $p<0.0001$ ). When both groups were compared there was highly significant statistical difference in mean heart rate between the two groups at 7 minutes after intubation.

The baseline values of SBP were comparable between groups with no significant difference.

Mean systolic blood pressure decreased by 3.96\% from baseline in Group E after induction of anaesthesia, which was statistically not significant ( $p>0.05$ ). In Group D mean systolic blood pressure increased by $0.77 \%$ from baseline, but the increase was not statistically significant ( $p>0.05$ ). There was no significant difference of mean systolic blood pressure between the two groups when compared after induction of anaesthesia.

At 1 minute after intubation, mean systolic blood pressure increased in both the groups from baseline (by $23.75 \%$ in Group E and by $3.80 \%$ in Group D). The increase was highly significant in Group E $(p<0.001)$ and the increase was not significant in Group D ( $p>0.05)$. The difference of mean systolic blood pressure between the two groups was highly significant $(\mathrm{p}<0.001)$ when compared at 1 minute after intubation.

Mean systolic blood pressure remained increased from baseline at 3 minutes after intubation in Group E. Statistical difference being highly significant $(\mathrm{p}<0.001)$. There was a decrease in mean systolic blood pressure by $4.41 \%$ in Group $\mathrm{D}$ at 3 minutes after intubation, which was statistically not significant $(p>0.05)$. The difference of mean systolic blood pressure between the two groups was highly significant $(\mathrm{p}<$ 0.001) when compared at 3 minutes after intubation [Figure 4].

At 5 minutes after intubation, the mean systolic blood pressure remained high when compared with baseline in Group E and it was highly significant ( $p<0.001)$. In Group D, mean systolic blood pressure was decreased by $8.56 \%$ and was highly significant $(\mathrm{p}<0.001)$. The difference of mean systolic blood pressure between the two groups was highly significant $(p<0.001)$ when compared at 5 minutes after intubation.

The mean systolic blood pressure in Group E remained high at 7 minutes after intubation when compared with baseline and was highly significant $(\mathrm{p}<0.001)$. The mean systolic blood pressure was decreased significantly $(p<0.05)$ in Group D when compared with baseline. The difference of mean systolic blood pressure between the two groups was highly significant $(p<0.001)$ when compared at 7 minutes after intubation.

The baseline values of DBP were comparable between two groups with no significant difference.

At 1 minute after intubation, there was increase in mean diastolic blood pressure in both the groups. The increase in mean diastolic pressure in Group E was highly significant when compared with the baseline mean diastolic blood pressure $(\mathrm{p}<0.001)$. The increase in mean diastolic blood pressure from baseline at 1 minute after intubation in Group D was not statistically significant ( $p>0.05$ ). When both the groups were compared at 1 minute after intubation, there 
was highly significant statistical difference of mean diastolic blood pressure between the two groups $(p=0.0001)$.

The mean diastolic blood pressure remained increased by 7.34\% from baseline in Group $\mathrm{E}$ at 3 minutes after intubation, which was statistically significant $(\mathrm{p}<0.05)$. There was a decrease in mean diastolic blood pressure by $4.43 \%$ from baseline in Group D at 3 minutes after intubation. When both groups were compared at 3 minutes after intubation there was highly significant statistical difference of mean diastolic blood pressure between the two groups $(\mathrm{p}<0.001)$.

The mean diastolic blood pressure decreased in both the groups at 5 minutes after intubation when compared with baseline. The decrease was not statistically significant in Group E ( $p>0.05)$, but the decrease was highly significant in Group D ( $p<0.001)$. When both the groups were compared with each other, there was highly significant statistical difference of mean diastolic blood pressure between the two groups at 5 minutes after intubation $(\mathrm{p}<0.001)$.

At 7 minutes after intubation, the mean diastolic blood pressure decreased in both groups when compared with the baseline values (4.79\% in Group E and $10.34 \%$ in Group D). The decrease being statistically insignificant in Group E ( $p>0.05)$, but was highly significant in Group D $(p<0.001)$. When both the groups were compared with each other, there was significant statistical difference of mean diastolic blood pressure between the two groups at 7 minutes after intubation $(\mathrm{p}<0.05)$.

The baseline values of MAP were comparable between two groups with no significant difference.

At 1 minute after intubation there was increase in mean MAP by $18.91 \%$ from baseline in Group E, which was highly significant $(\mathrm{p}<0.001)$. There was an increase in mean MAP in Group D from baseline (4.34\%), which was statistically insignificant $(\mathrm{p}>0.05)$. When both groups were compared there was a highly significant difference in mean MAP between the two groups $(p<0.001)$ at 1 minute after intubation [Table 6].

Mean MAP remained increased by $11.98 \%$ from baseline at 3 minutes in Group E, which was highly significant ( $p<0.001$ ). There was a decrease in mean MAP by $4.38 \%$ from baseline in Group D, but it was not statistically significant $(p>0.05)$. When both groups were compared, there was a highly significant difference in mean MAP between the two groups $(p<0.001)$ at 3 minutes after intubation [Figure 6].

At 5 minutes after intubation, mean MAP becomes comparable with baseline ( $p>0.05$ ) in Group E. There was a highly significant decrease in mean MAP (by $8.47 \%$ ) from baseline $(p<0.001)$ in Group D. There was a highly significant difference in mean MAP between the two groups when compared at 5 minutes after intubation $(p<0.001)$.

The mean MAP remained comparable with the baseline at 7 minutes after intubation in Group E ( $p>0.05)$. There was a highly significant decrease in mean MAP from baseline in Group D at 7 minutes after intubation. There was a highly significant difference in mean MAP between the two groups when compared at 7 minutes after intubation $(p<0.001)$.

One patient from Group E developed hypertension 1 minute after intubation. No episodes of bradycardia and hypotension were noted.

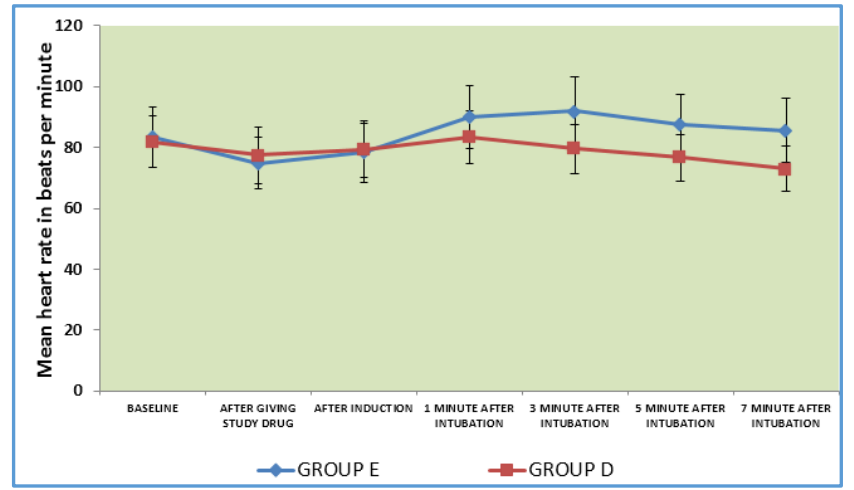

Figure 4

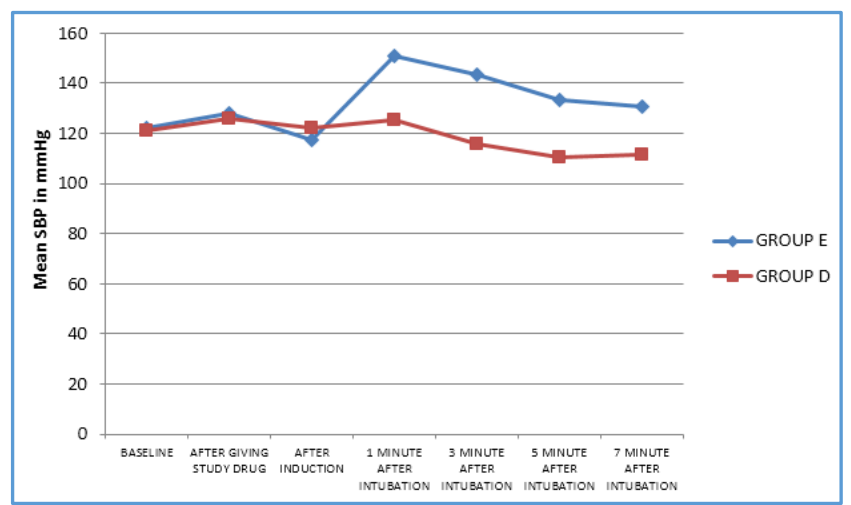

Figure 5

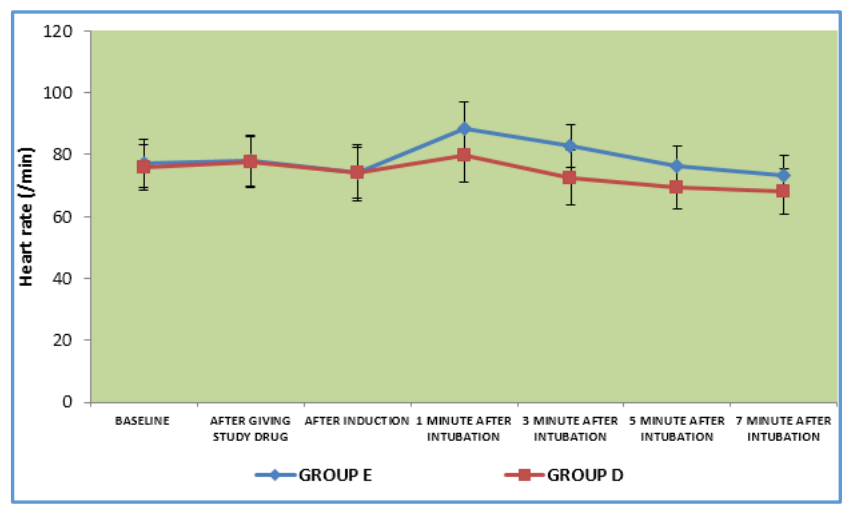

Figure 6

\section{DISCUSSION}

Maintaining intraoperative haemodynamic stability is of utmost importance in anaesthesia care. Stress produced by laryngoscopy and intubation produce undesirable haemodynamic effects in the form of tachycardia and hypertension is usually well tolerated in healthy individuals, but it can be deleterious in patient with hypertension, cardiac dysfunction, coronary artery disease and neurovascular disease. 4

Various pharmacological agents like nitroglycerine, 12 beta blockers, ${ }^{7}$ opioids, ${ }^{9}$ gabapentin, ${ }^{13}$ clonidine ${ }^{14}$ and dexmedetomidine 15 have been used to provide haemodynamic stability during perioperative period with varying success rate.

Esmolol is an ultra-short-acting cardio-selective betablocker which is commonly used for attenuation of intubation response in clinical practice. Among the $\beta$-adrenergic blocking drugs, esmolol seems to be an appropriate selection 
for attenuating the haemodynamic response to laryngoscopy and tracheal intubation because of its cardio-selectivity, rapid onset of action and short elimination half-life. ${ }^{16}$ There have been several reports discussing the effects of esmolol on both HR and arterial blood pressure during laryngoscopy and endotracheal intubation compared with placebo. ${ }^{5}$ Dexmedetomidine is a selective alpha- 2 agonist with alpha-2: alpha-1 activity of 1600:1. It was introduced in USA in clinical practice in 1999 and has been approved by FDA. In various studies, dexmedetomidine in a single pre-anaesthetic intravenous dose has been shown to reduce the anaesthetic requirement and blunt the haemodynamic response to stressful intraoperative events.

In the present study, we compared the effect of intravenous infusion of dexmedetomidine with that of esmolol on haemodynamic attenuation of pressor response to laryngoscopy and intubation. Demographic profiles like age, sex, weight, ASA physical status were comparable between the two groups $(p>0.05)$. The baseline haemodynamic parameters (mean HR, SBP, DBP, MAP) and mean duration of laryngoscopy were comparable in both the groups ( $p>0.05)$.

We measured and recorded baseline haemodynamic parameters (HR, SBP, DBP, MAP) of both the groups, after administration of study drug, after induction of anaesthesia and at 1 minute, 3 minutes, 5 minutes and 7 minutes after intubation. We compared the haemodynamic changes at different time interval between the two groups and with baseline.

The present study shows that the mean HR decreased in both the groups after infusion of study drug and was statistically significant in Group E ( $p<0.05)$.

After induction of anaesthesia the mean HR decreased in both the groups from baseline, but was statistically insignificant ( $p>0.05)$.

At 1 minute after intubation mean HR increased from baseline in both the groups, but the increase was more in Group E when compared with each other and it was statistically significant $(p<0.05)$. Moreover, the increase in mean HR in Group D was statistically insignificant ( $p>0.05)$.

At 3 minutes after intubation, mean HR in Group E remained significantly higher when compared to baseline, but the mean HR decreased in Group D when compared to baseline (statistically not significant).

At 5 and 7 minutes after intubation, the mean HR in Group E was comparable to baseline value ( $p>0.05)$. But there was a significant decrease in the mean HR from baseline in Group D. When both the groups were compared there was statistically significant difference between the two groups $(\mathrm{p}<0.05)$.

In pre-laryngoscopy period after infusion of the study drug there was an increase in mean SBP, mean DBP and mean MAP from baseline in both the groups. The increase in mean SBP after infusion of study drug in Group E was statistically significant $(p<0.05)$. After induction of anaesthesia, there was a decrease in mean SBP in Group E and an increase in mean SBP in Group D from baseline. And there was a decrease in mean DBP in both the groups after induction of anaesthesia. There was an increase in mean MAP after infusion of study drug in both the groups and there was a decrease in mean MAP in both the groups after induction of anaesthesia. These changes after induction of anaesthesia were not significant statistically ( $p>0.05$ ).
In the present study the maximum increase in mean SBP, DBP, MAP occurred at 1 minute and 3 minutes after intubation in Group E ( $p<0.05)$. The mean SBP, DBP and MAP increased in Group D at 1 minute after intubation, but it was not significant. When both the groups were compared, the increase in all the haemodynamic parameters studied at 1 minute and 3 minutes were significantly higher in Group E than in Group D and was significant statistically $(p<0.05)$.

At 5 minutes and 7 minutes after intubation, the mean SBP remained significantly raised from baseline in Group E, but the mean DBP and the mean MAP become comparable with the baseline. In Group D all the studied haemodynamic parameters decreased from baseline at 5 minutes and 7 minutes after intubation and it was statistically significant $(\mathrm{p}<0.05)$.

The incidence of hypertension and tachycardia following laryngoscopy and intubation were significantly lower in patients receiving intravenous dexmedetomidine infusion when compared with patients receiving intravenous esmolol infusion.

The only side effect observed was that of group Esmolol in the form of hypertension immediately after intubation. No other side effects were noted.

Yallapragada et al ${ }^{17}$ observed that intravenous dexmedetomidine is superior to intravenous esmolol in attenuating haemodynamic response to laryngoscopy and intubation, which is similar to our study result. In their study, they found that before induction HR decreased in groups, $23.7 \%$ in group dexmedetomidine and $12.1 \%$ in group esmolol from baseline as compared to $5.37 \%$ in Group D and $10.31 \%$ in Group E from baseline in our study. After induction of anaesthesia there was increase in heart rate in groups, $5.5 \%$ in group dexmedetomidine and $17.2 \%$ in group esmolol. At 1 minute after intubation there was increase in heart rate in both the groups, the increase being $0.9 \%$ in group dexmedetomidine and $18.7 \%$ in group esmolol from baseline compared to $1.90 \%$ in Group D and $7.66 \%$ in Group $\mathrm{E}$ in our study. At 3 minutes after intubation the mean heart rate significantly decreased in group dexmedetomidine, but was still increased significantly in group esmolol. At 5 minutes after intubation, heart rate become comparable to baseline in group Esmolol but significantly decreased in group Dexmedetomidine. At 1 minute after intubation the mean SBP, DBP and MAP increased in both the groups. In group Esmolol the increase in mean SBP, DBP and MAP were $24.9 \% 42.2 \% 29.6 \%$ respectively from baseline and in group dexmedetomidine the increase in mean SBP, DBP and MAP were $22.6 \%, 33.7 \%, 28.9 \%$ respectively from baseline. In our study the increase in mean SBP, DBP and MAP were less compared to this study. At 3 minutes after intubation also there was increase in mean SBP, DBP and MAP compared to baseline. At 5 minutes after intubation mean SBP, DBP and MAP remained increased by $1.9 \%, 11.2 \%$ and $7 \%$ respectively in group esmolol, but there was decrease in mean SBP by 11.85 and increase in mean DBP by $5.2 \%$ and increase in mean MAP by $0.1 \%$ respectively in group dexmedetomidine. The difference in change in percentage of mean HR, SBP, DBP and MAP in this study from our study may be due to the fact that they have used intravenous esmolol $0.5 \mathrm{mg} / \mathrm{kg}$ body weight and intravenous dexmedetomidine $0.5 \mathrm{mcg} / \mathrm{kg}$ body weight. 
Reddy SV et al ${ }^{18}$ compared the effect of dexmedetomidine $(1 \mathrm{mcg} / \mathrm{kg})$ and esmolol $(2 \mathrm{mg} / \mathrm{kg}$ ) in attenuating pressor response to laryngoscopy and intubation. In their study, they found that the increase in mean HR after intubation was seen in all the three groups. But the mean increase was minimal, $5.83 \%$ in Group dexmedetomidine ( $\mathrm{p}=0.0848)$ when compared with Group esmolol $14 \%(p=0.0152)$. Also, only in Group dexmedetomidine, there was no significant rise of HR at any time interval. The mean SBP, DBP and MAP were significantly lower in dexmedetomidine group than esmolol group. The results of this study correlate to our study.

Srivastava VK et $\mathrm{al}^{19}$ found that dexmedetomidine 1 $\mathrm{mcg} / \mathrm{kg}$ is more effective than esmolol for attenuating the haemodynamic response to laryngoscopy and intubation in elective neurosurgical patients. They found that after administration of the study drugs, decrease in HR were observed in group esmolol and group dexmedetomidine. Following induction, there was no difference in HR values between groups $D$ and $E(p=0.220)$, but HR values were significantly decreased in Group D and E ( $<<0.05)$ when compared with baseline values. Intubation caused an increase in the HR in both groups; however, the increase in group esmolol was more. The mean SBP and DBP values were lower in Group D after induction and all-time observation of intubation when compared with group esmolol and group control $(p<0.001)$. In group dexmedetomidine, there was no statistically significant increase in mean SBP and DBP, after intubation at any time intervals, while in group esmolol there was a statistically significant increase after intubation at 1, 2 and 3 minutes only. There was no significant increase in MAP comparative to baseline at any time intervals of intubation in group dexmedetomidine, while there was significant increase in group esmolol at 1, 2 and 3 minutes after intubation only ( $p<0.05)$. Results of this study correlated to our study.

Selvaraj et $\mathrm{al}^{20}$ in their study concluded that dexmedetomidine is more effective in attenuating the haemodynamic response to oral endotracheal intubation compared to that of esmolol hydrochloride. They found that both dexmedetomidine and esmolol produced a significant reduction in the values of the studied parameters (HR, SBP, DBP, MAP) after intubation. In dexmedetomidine group, there was a statistically significant decrease in all the studied parameters after intubation. However, in esmolol group, the decrease in DBP at 1 minute and 3 minutes after intubation were not statistically significant. In contrast to this study we found in our study that at 1 minute and 3 minutes after intubation in group esmolol there was significant increase in mean HR, SBP, DBP and MAP. This may be due to the fact that they used intravenous esmolol $0.5 \mathrm{mg} / \mathrm{kg}$ as slow intravenous injection over 30 seconds 2 minutes before intubation, whereas we used esmolol $1 \mathrm{mg} / \mathrm{kg}$ over 10 minutes infusion.

\section{The Limitations of Our Study are}

Firstly, the lack of placebo group is a limitation. However, we considered that tracheal intubation has been proven to produce an excessive sympathoadrenal response and furthermore would cause detrimental results even in normotensive patients.

Secondly, we have included only normotensives and the outcomes may not reflect the effectiveness and safety in hypertensive patients in whom attenuation of intubation response is more crucial. We sincerely think that attenuation of haemodynamic response to endotracheal intubation is also important in normotensives. Conducting the entire study in controlled hypertensives will be technically difficult to recruit patients as well to standardise the confounding factors such as drug therapy. For example, Angiotensin Converting Enzyme (ACE) inhibitors are one of the first line drugs in hypertensive management. Profound hypotension following anaesthetic induction in these patients with ACE inhibitors has been noted in previous studies. ${ }^{21,22}$ So inclusion of such patients will confound the haemodynamic response to intubation either with or without stopping the ACE inhibitor in the morning of surgery.

\section{CONCLUSION}

Evaluation of baseline and immediately after intubation values revealed a greater percentage variation in HR, SBP, DBP and MAP in the esmolol group as compared to the dexmedetomidine group.

Therefore, within the constraints of this study we demonstrated that administration of Dexmedetomidine $(1 \mu \mathrm{g} / \mathrm{kg})$ is a better agent than Esmolol $(0.5 \mathrm{mg} / \mathrm{kg})$ in attenuating the sympathomimetic response to laryngoscopy and intubation, whereas low dose Esmolol $(0.5 \mathrm{mg} / \mathrm{kg})$ is ineffective for the same purpose.

\section{REFERENCES}

[1] King BD, Harris LC Jr, Greifenstein FE, et al. Reflex circulatory responses to direct laryngoscopy and tracheal intubation performed during general anaesthesia. Anaesthesiology 1951;12(5):556-66.

[2] Pernerstorfer T, Kraffit P, Fitzgerald RD, et al. Stress response to tracheal Intubation: direct laryngoscopy compared with blind oral intubation. Anaesthesia 1995;50(1):17-22.

[3] Reid LC, Brace DE. Irritation of the respiratory tract and its reflex effect upon the heart. Surg Gynaec \& Obst 1940;70:157-62.

[4] Pyrs-Roberts C, Foex P, Biro GP, et al. Studies of anaesthesia in relation to hypertension. $\mathrm{V}$ : adrenergic Beta-receptor blockade. Br J Anaesth 1973;45(7):67181.

[5] Dyck JB, Maze M, Haack C, et al. Computer-controlled infusion of intravenous dexmedetomidine hydrochloride in adult human volunteers. Anaesthesiology 1993;78(5):821-8.

[6] Aantaa R, Jalonen J. Perioperative use of alpha-2 adrenoreceptor agonist and the cardiac patient. Eur J Anaesthesiol 2006;23(5):361-72.

[7] Venn RM, Bradshah CJ, Spencer R, et al. Preliminary UK experience of dexmedetomidine, a novel agent for postoperative sedation in the intensive care unit. Anaesthesia 1999;54(12):1136-42.

[8] Williams PL, Warwick R. Gray's anatomy. 36 th edn. Edinburgh: Churchill Livingstone 1985.

[9] Sharma S, Mitra S, Grover VK, et al. Esmolol blunts the haemodynamic responses to tracheal intubation in treated hypertensive patients. Can J Anaesth 1996;43(8):778-82. 
[10] Zargar JA, Naqash IA, Gurcoo SA, et al. Comparative evaluation of the effect of metoprolol and esmolol on rate pressure product and ECG changes during laryngoscopy and endotracheal intubation in controlled hypertensive patients. Indian J Anesth 2002;46(5):365-8.

[11] Oxorn D, Knox JW, Hill J. Bolus doses of esmolol for the prevention of perioperative hypertension and tachycardia. Can J Anaesth 1990;37(2):206-9.

[12] Greenspan AM, Spielman SR, Horowitz LN, et al. Electrophysiology of esmolol. Am J Cardiol 1985;56(11):19F-26F.

[13] Elkayam U, Aronow WS. Glyceryl trinitrate (Nitroglycerine) ointment and isosorbide dinitrate: review of their pharmacological properties and therapeutic use. Drugs 1982;23(3):165-94.

[14] Stoelting RK. Circulatory changes during direct laryngoscopy and tracheal intubation: influence of duration of laryngoscopy with or without prior lidocaine. Anaesthesiology 1977;47(4):381-4.

[15] Vickery RG, Sheridan BC, Sega IS, et al. Anesthetic and hemodynamic effects of the stereoisomers of medetomidine, an alpha 2-adrenergic agonist, in halothane-anesthetized dogs. Anesth Analg 1988;67(7):611-5.

[16] Kovac AL, Masiongale A. Comparison of nicardipine versus esmolol in attenuating the hemodynamic responses to anesthesia emergence and extubation. J Cardiothorac Vasc Anesth 2007;21(1):45-50.
[17] Yavascaoglu B, Kaya FN, Baykara M, et al. A comparison of esmolol and dexmedetomidine for attenuation of intraocular pressure and haemodynamic responses to laryngoscopy and tracheal intubation. Eur J Anaesthesiol 2008;25(6):517-9.

[18] Reddy SV, Balaji D, Ahmed SN. Dexmedetomidine versus esmolol to attenuate the hemodynamic response to laryngoscopy and tracheal intubation: a randomized double-blind clinical study. Int J App Basic Med Res 2014;4(2):95-100.

[19] Srivastava VK, Agrawal S, Gautam SK, et al. Comparative evaluation of esmolol and dexmedetomidine for attenuation of sympathomimetic response to laryngoscopy and intubation in neurosurgical patients. J Anaesthesiol Clin Pharmacol 2015;31(2):186-90.

[20] Selvaraj V, Manoharan KR. Prospective randomized study to compare between intravenous dexmedetomidine and esmolol for attenuation of hemodynamic response to endotracheal intubation. Anesth Essays Res 2016;10(2):343-8.

[21] Bertrand M, Goger G, Meersschaert K, et al. Should the angiotensin II antagonist be discontinued before surgery? Anesth Analg 2001;92(1):26-30.

[22] Rajgopal R, Rajan S, Sapru K, et al. Effect of preoperative discontinuation of angiotensin converting enzymes inhibitors or angiotensin II receptor antagonists on intraoperative arterial pressures after induction of general anaesthesia. Anesth Essay Res 2014;8(1):32-5. 